\title{
Theoretical observers for infinite dimensional skew-symmetric systems
}

\author{
Deguenon Judicael and Alina Barbulescu
}

\begin{abstract}
The observer construction has a main importance in the control theory and its applications for the systems of infinite dimension. Even if the system' state has an infinite dimension, its estimation is given using some physical measures of finite dimensions. Considering unbounded boundary observations operators and assuming that the exact observability property holds, we build some Luenberger like observers which assure the exponential stability of the error system under some regularity conditions.
\end{abstract}

\section{Introduction}

The observer construction has a main importance in the control theory and its applications for the systems of infinite dimension. Even if the state of system has an infinite dimension, its estimation is given using some physical measures of finite dimesions.

Systems with bounded input and output operators have been studied in [1], [3], [9]. As presented in [7] there are three different classes of systems: (a) the Pritchard-Salamon class [12], [14]; (b) the Weiss class of regular systems [2], and [18] and (c) the Salamon class of well-posed linear systems [15] and [16].

The complexity of the situation in infinite dimension by comparison to that in the finite one is summarized in [7] and appears because of the high gain that can produce the instability of the error' system.

Key Words: observability, operator, regularity, stability

2010 Mathematics Subject Classification: 93B05, 93B07

Received: 07.04.2019

Accepted: 10.05.2019 
This part contain an overview of the basic notions necessary for the proof of the main result of this article, while the main result of this paper, presented in the next section, is related to the collocated feedback exponential stabilization [5], [6], [17], [22], [23].

Assume that a linear infinite dimensional skew-adjoint observation system is defined on the Hilbert space $X$ and the observation space is another Hilbert space $O$. Considering unbounded boundary observations operators and assuming that the exact observability property holds, we build some Luenberger like observers which assure the exponential stability of the error system under some regularity conditions.

Let $X$ be a Banach space and $I$ the identity on $X$.

Definition 1. [11] A $C^{o}$ semigroup of operators is a family of linear operators from $X$ to $X, T(t)_{t \geq 0}$ satisfying:

i) $T(0)=I$,

ii) $T(t) T(\tau)=T(t+\tau), \forall t, \tau \geq 0$.

ii) $\lim _{t \rightarrow 0^{+}} T(t) \phi=\phi, \forall \phi \in X$.

The domain of definition of an operator $A$ will be denoted by $D(A)$.

Definition 2. [11] A generator of the semigroup $T(t)_{t \geq 0}$ is an operator $A$ defined by the equation:

$$
A \phi=\lim _{h \rightarrow 0^{+}} \frac{T(h) \phi-\phi}{h},
$$

where the limit is evaluated in terms of the norm on $X$ and $\phi \in D(A)$ iff this limit exists.

Theorem 1 [11] Let $T(t)_{t \geq 0}$ be a $C^{o}$ semi-group on $X, A$ its generator and $\phi \in D(A)$. Then:

1) $T(t) \phi \in D(A)$ for all $t \geq 0$ and $\frac{d}{d t} T(t) \phi=A T(t) \phi=T(t) A \phi$.

2) $A$ is a closed operator, whose domain is dense on $X$.

3) There are two contants $M \geq 1$ and $\omega \in \mathbf{R}$ such that $\|T(t)\| \leq M e^{\omega t}, \forall t \geq 0$.

Definition 3. [4] Let $T(t)_{t>0}$ be a $C^{o}$ semigroup on $X, A$ its generator and $\phi \in D(A)$. The number defined by $\omega_{0}(A)=\inf \left\{\omega / \exists M,\|T(t)\| \leq M e^{\omega t}, \forall t \geq\right.$ $0\}$ is called the exponentially increasing rate of of $T(t)$. If $\omega<0$ we say that the semigroup $T(t)_{t \geq 0}$ is exponentially stable.

Definition 4. [11] A $C^{0}$ group of bounded linear operators on $X$ is a family $(T(t))_{t \in \mathbf{R}}$ of operators on $X$, such that:

i) $T(0)=I$.

ii) $T(t) T(\tau)=T(t+\tau), \forall t, \tau \in \mathbf{R}$.

ii) $\lim _{t \rightarrow 0} T(t) \phi=\phi, \forall \phi \in X$. 
Theorem 2(Stone) [11] Let $X$ be a Hilbert space. $A$ is the generator of a group of unity operators on $X$ iff $A$ is anti-adjoint. Consider a distributed non - excited system [8]:

$$
\left(\sum\right)\left\{\begin{aligned}
\dot{\phi}(t) & =A \phi(t), \forall t \geq 0 \\
\phi(0) & =\phi_{0}
\end{aligned}\right.
$$

Suppose that we collect $q$ measures on the system, defined by the output function:

$$
(S)\left\{\begin{aligned}
y(t) & =\left(y_{1}(t), y_{2}(t), \ldots, y_{q}(t)\right) \\
& =C \phi(t)
\end{aligned}\right.
$$

where $C$ is an unbounded operator, whose domain, $D(C) \subset X$ is invariant with respect to the $C^{0}$ semigroup $T(t)_{t \geq 0}$ and $y(.) \in L^{2}\left(0, T ; \mathbf{R}^{q}\right)$.

Definition 5(exact observability) [13] The system $\left(\sum\right)$ together with $(S)$ is exactly observable if there are constants $\tau_{0}>0$ and $M>0$ such that:

$$
M^{-1}\left\|\phi_{0}\right\|_{X}^{2} \leq \int_{0}^{\tau_{0}}\left\|C T(t) \phi_{0}\right\|_{O}^{2} d t \leq M\left\|\phi_{0}\right\|_{X}^{2} .
$$

Let $X$ be the state space, $U$ the input space, $O$ the output space. Suppose that $X, U$ and $O$ are Hilbert spaces, with their inner products. Consider, in infinite dimension, the time invariant linear system described by [19]:

$$
\text { (П) }\left\{\begin{aligned}
\dot{\phi}(t) & =A \phi(t)+B u(t), \\
y(t) & =C \phi(t)+D u(t), \\
\phi(0) & =\phi_{0} .
\end{aligned}\right.
$$

$\phi_{0}$ is called the initial state of the system (П).

$\phi(t) \in X$ is called the state of system $\left(\prod\right)$ at the moment $t$.

$u(t) \in L^{2}([0, \infty), U)$ is the control and $y(t) \in L^{2}([0, \infty), O)$ is the output.

$A$ is generally an unbounded operator, generator of a $C^{0}$ semigroup on $X$.

Let $\rho(A)$ be the resolvent set of $A$ and $\beta \in \rho(A)$. We denote by $X_{1}$, the domain $D(A)$, with the norm $\|\varphi\|_{1}=\|(\beta I-A) \varphi$. The closure of $X$, with the norm $\|\varphi\|_{-1}=\left\|(\beta I-A)^{-1} \varphi\right\|_{X}$ will be denoted by $X_{-1}$.

So $X_{1} \subset X \subset X_{-1}$.

We consider the extension of $A$ such that $A \in L\left(X, X_{-1}\right)$ and the extension of the semigroup $(T(t))_{t \geq 0}$ on $X_{-1}$. For all $\beta \in \rho(A),(\beta I-A)^{-1}$ can be extended to the isometric isomorphism from $X_{-1}$ to $X$.

We shall denote the operators and their extensions by the same symbols.

$B$ is called control operator, $B \in L\left(U, X_{-1}\right)$. 
We asume that $B$ is bounded if $B \in L(U, X)$ and unbounded if $B \notin$ $L(U, X)$.

$C \in L\left(X_{1}, O\right)$ is called output operator.

We denote by $C_{\Lambda}$ the $\Lambda$ - extension of $\mathrm{C}$, defined by:

$$
\left\{\begin{aligned}
D\left(C_{\Lambda}\right) & =\left\{x \in X, \lim _{\lambda \rightarrow+\infty} \lambda C(\lambda I-A)^{-1} x \text { exists }\right\} \\
C_{\Lambda} x & =\lim _{\lambda \rightarrow+\infty} \lambda C(\lambda I-A)^{-1} x, \forall x \in D\left(C_{\Lambda}\right) .
\end{aligned}\right.
$$

Let $\lambda_{0} \in \mathbf{R}$ such that $\left[\lambda_{0}, \infty\right) \subset \rho(A)$. We define the norm on $D\left(C_{\Lambda}\right)$ :

$$
\|x\|_{D\left(C_{\Lambda}\right)}=\|x\|_{X}+\sup _{\lambda \geq \lambda_{0}}\left\|\lambda C(\lambda I-A)^{-1} x\right\|_{O} .
$$

Endowed with this norm, $D\left(C_{\Lambda}\right)$ is a Banach space.

$C_{\Lambda} \in L\left(D\left(C_{\Lambda}\right), O\right), X_{1} \subset D\left(C_{\Lambda}\right) \subset X$ with the continuous injection and $X_{1}$ is dense in $D\left(C_{\Lambda}\right)$.

$\mathrm{D}$ is the feedthrough operator of $\mathrm{G}$ and $D \in L(U, O) . G$ is the transfer function of $\left(\prod\right)$.

If $u=0,\left(\prod\right)$ is called open loop system and will be denoted by $\left(\prod^{0}\right)$.

Assume that $u \neq 0$ in $\left(\prod\right)$.

Definition 6. [20] $B$ is called an admissible control operator for the semigroup $T(t)_{t \geq 0}$, if there is $\tau>0$ such that $\Phi_{\tau} u \in X, \forall u \in L^{2}([0, \infty), U)$, where $\Phi_{\tau} u$ is defined by

$$
\Phi_{\tau} u=\int_{0}^{\tau} T(\tau-\sigma) B u(\sigma) d \sigma .
$$

Proposition 1. [20] If $B$ is an admissible operator for the semigroup $(T(t))_{t \geq 0}$, then there is $k \geq 0$, such that for any $s \in \mathbf{C}_{0}$, big enough:

$$
\left\|(s I-A)^{-1} B\right\|_{L(U, X)} \leq k / \sqrt{\Re e(s)},
$$

where $\Re e(s)$ is the real part of $s$.

Definition 7. [20] The system (П) or the quadruple $(A, B, C, D)$ is (Weiss) regular if:

i) The couples $(A, C)$ and $(A, B)$ are admissible.

ii) $\operatorname{Im}(\lambda I-A)^{-1} B \subset D\left(C_{\Lambda}\right), \forall \lambda \in \rho(A)$.

iii) The transfer function $C_{\Lambda}(s I-A)^{-1} B$ is analytic and uniformly bounded on a certain $\mathbf{C}_{\alpha}$.

iv) The input-output transfer function $G(s)=C_{\Lambda}(s I-A)^{-1} B+D\left(s \in \mathbf{C}_{\alpha}\right)$ is regular, that is $\forall v \in U, \exists \lim _{\mathbf{R} \ni \lambda \rightarrow+\infty} G(\lambda) v=D v$, where $D$ is the feedthrough 


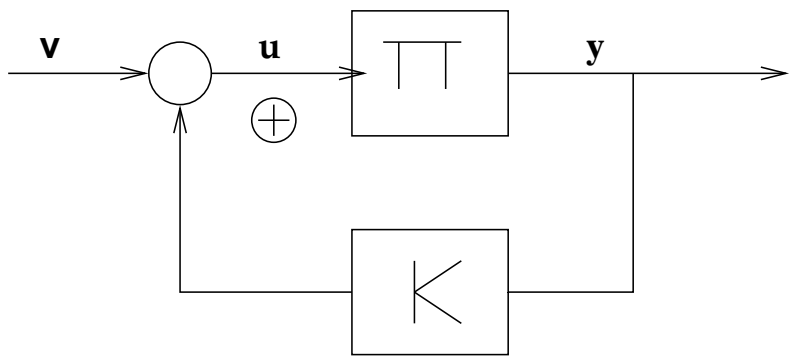

Figure 1: Closed loop system $\prod^{K}$

operator of $G$.

In other words:

$$
\lim _{\mathbf{R} \ni \lambda \rightarrow+\infty} C_{\Lambda}(\lambda I-A)^{-1} B v=0, \quad v \in U .
$$

Theorem 3 [20] If $\left(\prod\right)=(A, B, C, D)$ is a linear regular system, then, for all $\phi_{0} \in X$ and for all $u \in L_{l o c}^{2}([0, \infty) ; U)$, the system:

$$
\left\{\begin{aligned}
\dot{\phi}(t) & =A \phi(t)+B u(t) \\
y(t) & =C_{\Lambda} \phi(t)+D u(t) \\
\phi(0) & =\phi_{0},
\end{aligned}\right.
$$

admits an unique strong solution $\phi(t)=T(t) \phi_{0}+\int_{0}^{t} T(t-\tau) B u(\tau) d \tau$ satisfying $\phi(0)=\phi_{0}$. Moreover, if $u$ and $y$ are continuous to the right for all $t \geq 0$, then $\phi(t) \in D\left(C_{\Lambda}\right)$.

Assume that the system $\left(\prod\right)$ is in a loop, with the feedback law: $u(t)=$ $K y(t)+v(t)$ where $K$ is the output feedback operator, i.e. $K \in L(O, U)$ and $v($.$) is a new input (Fig.1).$

Definition 8 [19] Let $\widetilde{G}(s)$ be a well - posed transfer function and $K \in$ $L(O, U) . K$ is an admissible output feedback operator for $\widetilde{G}(s)$ if $I-K \widetilde{G}(\cdot)$ is invertible on $H_{\infty}^{\infty}(L(U))$, i.e. there is $\alpha \in \mathbf{R}$ such that $I-K \widetilde{G}(s)$ is invertible for all $s \in \mathbf{C}_{\alpha}$ and the inverse $(I-K \widetilde{G}(s))^{-1}$ is analytic and uniformly bounded on $\mathbf{C}_{\alpha}$.

Proposition 2 [19] Assume that the transfer $G$ is regular and the feedthrough operator $D \in L(U, O)$ satisfies: $\lim _{\sigma \rightarrow+\infty} \sup _{\delta \in \mathbf{R}}\|G(\sigma+i \delta)-D\|=0$. Then, 
for all $K \in L(O, U), K$ is an admissible feedback operator iff $I-D K$ is invertible.

Assume that the open loop system $\left(\prod\right)$ is regular and $\mathrm{K}$ is an admissible output feedback operator such that the closed loop system $\left(\Pi^{K}\right)$ is also regular. Then, the closed loop system $\left(\prod^{K}\right)$ is described by the system:

$$
\left(\prod^{K}\right):\left\{\begin{aligned}
\dot{\phi}(t) & =A^{K} \phi(t)+B^{K} u(t), \\
y(t) & =C^{K} \phi(t)+D^{K} u(t), \\
\phi(0) & =\phi_{0}
\end{aligned}\right.
$$

where: $D\left(C_{\Lambda}^{K}\right)=D\left(C_{\Lambda}\right), C_{\Lambda}^{K}=(I-D K)^{-1} C_{\Lambda}, B^{K}=B(I-D K)^{-1}$,

$$
\left\{\begin{aligned}
D\left(A^{K}\right) & =\left\{x \in D\left(C_{\Lambda}\right),\left(A+B K(I-D K)^{-1} C_{\Lambda}\right) x \in X\right\}, \\
A^{K} x & =\left(A+B K(I-D K)^{-1} C_{\Lambda}\right) x, \forall x \in D\left(A^{K}\right), \\
C^{K} x & =(I-D K)^{-1} C_{\Lambda} x, \forall x \in D\left(A^{K}\right) .
\end{aligned}\right.
$$

Theorem 4 [19] If ( $\prod$ ) is regular, $\mathrm{K}$ admissible, $I-D K$ invertible, then $\left(\prod^{K}\right)$ is regular and

$$
G^{K}(s)=(I-G(s) K)^{-1} G(s), D^{K}=(I-D K)^{-1} D .
$$

Remark 1 [19], [21] If ( $\prod$ ) is observable and $K$ is admissible, then $\left(\prod^{K}\right)$ is observable.

\section{Main Result}

In this chapter we work in the general theoretical frame. We consider the linear autonomous system, observed on the state space $X$, supposed to be a Hilbert space:

$$
\left\{\begin{aligned}
\dot{\phi}(t) & =A \phi(t) \\
y(t) & =C \phi(t) \\
\phi(0) & =\phi_{0}
\end{aligned}\right.
$$

where $A$ is the generator of a $C^{0}$ group of unity operators on $X, C: X_{1} \rightarrow O$ is a linear bounded operator, $X_{1}$ being the Banach space $D(A)$, endowed with the norm: $\|\varphi\|_{X_{1}}=\|(\beta I-A) \varphi\|_{X}$, with $\beta \in \rho(A) \cap \rho(-A)$.

The Hilbert spaces $X$ and $O$ are identified respectively with their topological duals, $X^{\prime}$ and $O^{\prime}$. If $X_{-1}$ is the topological dual of $X_{1}$, the duality product on $X_{1} \times X_{-1}$, denoted by $<., .>_{X_{1} \times X_{-1}}$, is defined as the continuous extension of the inner product on $X$ :

$$
<\varphi, f>_{X_{1} \times X_{-1}}=<\varphi, f>_{X}, \quad \forall \varphi \in X_{1}, f \in X .
$$


We also have the following continuous and dense injections: $X_{1} \subset X \subset X_{-1}$.

The dual space $X_{-1}$ is also a Hilbert space with the induced norm:

$$
\|\varphi\|_{X_{-1}}=\left\|(\beta I+A)^{-1} \varphi\right\|_{X} .
$$

Moreover, $(\beta I-A) \in L\left(X_{1}, X\right)$ and $(\beta I+A) \in L\left(X, X_{-1}\right)$ are isometric isomorphisms.

The group $\left(e^{t A}\right)_{t \in \mathbf{R}}$ generated by $A$ can be extended to a $C^{0}$ semigroup on $X_{-1}$. If $C^{*}$ denotes the adjoint operator of $C$, then $C^{*} \in L\left(O, X_{-1}\right)$.

We also suppose that $(A, C)$ is exactly observable.

The observer proposed by us is described by the system:

$$
\dot{\psi}(t)=\left[A-\kappa C^{*} C_{\Lambda}\right] \psi(t)+\kappa C^{*} y(t), \quad \kappa>0, \psi(0)=\psi_{0} .
$$

Let denote by $A^{\kappa}=A-\kappa \cdot C^{*} C_{\Lambda}$ and $\varepsilon(t)=\psi(t)-\phi(t)$.

Consider that the estimation error satisfies the evolution equation:

$$
\dot{\varepsilon}(t)=A^{\kappa} \varepsilon(t), \kappa>0, \varepsilon(0)=\varepsilon_{0} .
$$

and the auxiliary system:

$$
\dot{\Omega}(t)=A \Omega(t)+C^{*} v(t), z(t)=C_{\Lambda} \Omega(t) .
$$

Definition 9 The observer (7) is said to be (exponentially) convergent or stable if (9) is regular and (8) is exponentially stable.

In the following we shall prove the main result:

Theorem 5 Let $A$ be a generator of a $C^{0}$ group of unity operators on $X$. If $\left(A, C^{*}, C\right)$ is regular and $(A, C)$ is exactly observable, then the observer (7) has an unique solution on $C([0, \infty), X)$ for all $\left(\phi_{0}, \psi_{0}\right) \in X \times X$ and its state is exponentially convergent on $X$ to the state of the system (6), for all $0<\kappa<1 / K_{\max }$. The observer (7) is exponentially instable if $\kappa>1 / K_{\min }$, where:

$$
\begin{aligned}
K_{\text {max }}= & \sup _{\left|C_{\Lambda} f\right|_{O}=1} \varlimsup_{\substack{\beta \in \mathbf{R}^{+} \\
\beta \rightarrow+\infty}} \beta\left\|(\beta I-A)^{-1} C^{*} C_{\Lambda} f\right\|_{X}^{2}, \\
K_{\min }= & \inf _{\left|C_{\Lambda} f\right|_{O}=1} \underline{\lim } \underset{\beta}{\beta \rightarrow \mathbf{R}^{+}} \underset{\beta \rightarrow+\infty}{ } \beta\left\|(\beta I-A)^{-1} C^{*} C_{\Lambda} f\right\|_{X}^{2} .
\end{aligned}
$$

Proof. For simplicity, we consider $X$ as a real Hilbert space. The same results are true if $X$ is a complex Hilbert space, after a slight modification of the proof. 
Step I. We prove that the observer (7) admits an unique solution on $C([0, \infty), X)$.

By hypothesis, $\left(A, C^{*}, C\right)$ is regular (with the null feedthrough operator).

Let $G(s)$ be the tranfer function of $\left(A, C^{*}, C_{\Lambda}\right)$ representing the auxiliary system (9).

By regularity, $G(s)=C_{\Lambda}(s I-A)^{-1} C^{*} \in H^{\infty}\left(\mathbf{C}_{\alpha}, L(O)\right)$ for a certain $\alpha>0$ and

$$
\lim _{s \rightarrow+\infty} G(s) v=0, \forall v \in O .
$$

It also results that the feedthrough operator is null for the auxiliary system (9).

Definition 10 Let $\widetilde{G}(s): U \rightarrow U$ be a transfer function such that $\widetilde{G} \in$ $H^{\infty}\left(\mathbf{C}_{0}\right) . \widetilde{G}(s)$ is said to be a real positive transfer function if $\widetilde{G}(s)+\widetilde{G}(s)^{*} \geq 0$ for all $s \in \mathbf{C}_{0}$.

Assertion 1 [24] The transfer function of the system (9) is real positive.

Assertion 2 If $\widetilde{G}(s)$ is a real positive transfer function, then, for each $\kappa>0$, the output feedback operator $K=-\kappa I$ is admisible for $\widetilde{G}(s)$.

Proof of Assertion 2 It is known that [21]: if $c I+\widetilde{G}(s)$ is a real positive transfer function for a certain $c \geq 0$, then, for any $k \in(0,1 / c)$, the operator $K=-k I$ is admissible for $\widetilde{G}(s)$.

In particular, for $c=0$ we obtain that $K=-k I$ is admissible for $\widetilde{G}(s)$, $\forall k>0$.

The assertion 1 is proved.

From Assertion 2 it results that any output feedback operator $K=-\kappa I$, $\kappa>0$, is admissible.

From Theorem 4 it results that the closed loop system:

$$
\left\{\begin{aligned}
\dot{\Omega}(t) & =\left[A-\kappa C^{*} C_{\Lambda}\right] \Omega(t)+\kappa \xi(t) \quad, \kappa>0 \\
z(t) & =C_{\Lambda} \Omega(t)
\end{aligned}\right.
$$

obtained by the feedback $v(t)=K z(t)+\kappa \xi(t)$ is also regular, with the null feedthrough.

If $\xi(t)=y(t)$, the closed loop system (13) is the observer (7). From Theorem 3 and (4), it results that $A^{\kappa}$ is the generator of a $C^{0}$ closed loop semigroup and is defined by:

$$
\left\{\begin{aligned}
D\left(A^{\kappa}\right) & =\left\{\varphi \in D\left(C_{\Lambda}\right) /\left(A-\kappa C^{*} C_{\Lambda}\right) \varphi \in X\right\} \\
A^{\kappa} \varphi & =\left(A-\kappa C^{*} C_{\Lambda}\right) \varphi, \quad \forall \varphi \in D\left(A^{\kappa}\right)
\end{aligned}\right.
$$


Moreover, the system ( 7$)$ is regular, and $\forall\left(\phi_{0}, \psi_{0}\right) \in X \times X, y \in L_{l o c}^{2}([0, \infty), O)$, $\psi \in C([0, \infty), X)$, with $\psi(t)=e^{t A_{\kappa}} \psi_{0}+\kappa \int_{0}^{t} e^{(t-\tau) A_{\kappa}} C^{*} C_{\Lambda} e^{\tau A} \phi_{0} d \tau$.

The first step is completed.

Step II. The error estimation.

Assertion 3 For any $\varepsilon(0) \in D\left(A^{\kappa}\right)$, the solution of the system satisfies the equalities:

$$
\begin{aligned}
\frac{1}{2} \frac{d}{d t}\|\varepsilon(t)\|_{X}^{2} & =<A^{\kappa} \varepsilon(t), \varepsilon(t)>_{X} \\
& =-\kappa\left\|C_{\Lambda} \varepsilon(t)\right\|_{O}^{2}+\lim _{\beta \rightarrow+\infty} \kappa^{2} \beta\left\|R(\beta, A) C^{*} C_{\Lambda} \varepsilon\right\|_{X}^{2}
\end{aligned}
$$

Proof of Assertion 3 The identity (15) can be easily obtained.

To prove (16), remember that $\forall \lambda \in \rho(A), R(\lambda, A)$ is an isomorphism from $X$ to $X_{-1} ; R(\lambda, A)$ commutes with $A$ on $D(A)$ and:

$$
\lim _{\lambda \rightarrow \infty} \lambda R(\lambda, A) x=x, \quad \lim _{\lambda \rightarrow \infty} \lambda R(\lambda,-A) x=x \quad \forall x \in X .
$$

Let fix $\beta \in \rho(A) \cap \mathbf{R}^{+}$. Then:

$$
\begin{gathered}
\varepsilon+R(\beta, A) \kappa C^{*} C_{\Lambda} \varepsilon=R(\beta, A)\left[\beta \varepsilon-A^{\kappa} \varepsilon\right] \in D(A), \quad \forall \varepsilon \in D\left(A^{\kappa}\right) \Leftrightarrow \\
A^{\kappa} \varepsilon=A\left[\varepsilon+R(\beta, A) \kappa C^{*} C_{\Lambda} \varepsilon\right]-\beta R(\beta, A) \kappa C^{*} C_{\Lambda} \varepsilon, \quad \forall \varepsilon \in D\left(A^{\kappa}\right) .
\end{gathered}
$$

Passing to the inner product on $X$, we obtain:

$$
\begin{gathered}
\left\langle A^{\kappa} \varepsilon, \varepsilon\right\rangle_{X}=\left\langle A\left[\varepsilon+R(\beta, A) \kappa C^{*} C_{\Lambda} \varepsilon\right], \varepsilon\right\rangle_{X}-\left\langle\beta R(\beta, A) \kappa C^{*} C_{\Lambda} \varepsilon, \varepsilon\right\rangle_{X}= \\
=\left\langle A\left[\varepsilon+R(\beta, A) \kappa C^{*} C_{\Lambda} \varepsilon\right],\left[\varepsilon+R(\beta, A) \kappa C^{*} C_{\Lambda} \varepsilon\right]-R(\beta, A) \kappa C^{*} C_{\Lambda} \varepsilon\right\rangle_{X}- \\
-\left\langle\beta R(\beta, A) \kappa C^{*} C_{\Lambda} \varepsilon, \varepsilon\right\rangle_{X}= \\
=\left\langle A\left[\varepsilon+R(\beta, A) \kappa C^{*} C_{\Lambda} \varepsilon\right],\left[\varepsilon+R(\beta, A) \kappa C^{*} C_{\Lambda} \varepsilon\right]\right\rangle_{X}- \\
-\left\langle A\left[\varepsilon+R(\beta, A) \kappa C^{*} C_{\Lambda} \varepsilon\right), R(\beta, A) \kappa C^{*} C_{\Lambda} \varepsilon\right\rangle_{X}-\left\langle\beta R(\beta, A) \kappa C^{*} C_{\Lambda} \varepsilon, \varepsilon\right\rangle_{X}
\end{gathered}
$$

Since $A$ is anti - adjoint, the first term in the right - hand side is null, so

$$
\begin{aligned}
\left\langle A^{\kappa} \varepsilon, \varepsilon\right\rangle_{X}= & -\left\langle A\left[\varepsilon+R(\beta, A) \kappa C^{*} C_{\Lambda} \varepsilon\right], R(\beta, A) \kappa C^{*} C_{\Lambda} \varepsilon\right\rangle_{X} \\
& -\left\langle\beta R(\beta, A) \kappa C^{*} C_{\Lambda} \varepsilon, \varepsilon\right\rangle_{X} .
\end{aligned}
$$

By (17), and since $A$ is anti-adjoint on $X$ we obtain,

$$
\begin{aligned}
& -\left\langle A\left[\varepsilon+R(\beta, A) \kappa C^{*} C_{\Lambda} \varepsilon\right], R(\beta, A) \kappa C^{*} C_{\Lambda} \varepsilon\right\rangle_{X}= \\
= & -\lim _{\lambda \rightarrow+\infty}\left\langle A\left[\varepsilon+R(\beta, A) \kappa C^{*} C_{\Lambda} \varepsilon\right], \lambda R(\lambda, A) R(\beta, A) \kappa C^{*} C_{\Lambda} \varepsilon\right\rangle_{X}= \\
= & \lim _{\lambda \rightarrow+\infty}\left\langle\left[\varepsilon+R(\beta, A) \kappa C^{*} C_{\Lambda} \varepsilon\right],-\lambda A R(\lambda, A) R(\beta, A) \kappa C^{*} C_{\Lambda} \varepsilon\right\rangle_{X} .
\end{aligned}
$$


$A$ commutes with $R(\lambda, A)$. Therefore:

$$
-\lambda A R(\lambda, A) R(\beta, A) \kappa C^{*} C_{\Lambda} \varepsilon=-\lambda R(\lambda, A)[A R(\beta, A)] \kappa C^{*} C_{\Lambda} \varepsilon .
$$

From the identity $(\beta I-A) R(\beta, A)=I$, it results:

$$
A R(\beta, A)=-I+\beta R(\beta, A) .
$$

From (20), (19) we deduce that:

$$
\begin{aligned}
& -\left\langle A\left[\varepsilon+R(\beta, A) \kappa C^{*} C_{\Lambda} \varepsilon\right],-R(\beta, A) \kappa C^{*} C_{\Lambda} \varepsilon\right\rangle_{X} \\
= & \lim _{\lambda \rightarrow+\infty}\left\langle\left[\varepsilon+R(\beta, A) \kappa C^{*} C_{\Lambda} \varepsilon\right],-\lambda R(\lambda, A) \kappa C^{*} C_{\Lambda} \varepsilon\right. \\
& \left.+\beta R(\beta, A) \lambda R(\lambda, A) \kappa C^{*} C_{\Lambda} \varepsilon\right\rangle_{X} .
\end{aligned}
$$

Also,

$$
-\left\langle\beta R(\beta, A) \kappa C^{*} C_{\Lambda} \varepsilon, \varepsilon\right\rangle_{X}=-\lim _{\lambda \rightarrow+\infty}\left\langle\beta R(\beta, A) \lambda R(\lambda, A) \kappa C^{*} C_{\Lambda} \varepsilon, \varepsilon\right\rangle_{X}
$$

Replacing (22), (21) in (18), we obtain

$$
\begin{aligned}
\left\langle A^{\kappa} \varepsilon, \varepsilon\right\rangle_{X} & =\lim _{\lambda \rightarrow+\infty}\left\langle\varepsilon,-\lambda R(\lambda, A) \kappa C^{*} C_{\Lambda} \varepsilon\right\rangle_{X}+ \\
& +\lim _{\lambda \rightarrow+\infty}\left\langle R(\beta, A) \kappa C^{*} C_{\Lambda} \varepsilon,-\lambda R(\lambda, A) \kappa C^{*} C_{\Lambda} \varepsilon\right\rangle_{X}+ \\
& +\lim _{\lambda \rightarrow+\infty}\left\langle R(\beta, A) \kappa C^{*} C_{\Lambda} \varepsilon, \beta R(\beta, A) \lambda R(\lambda, A) \kappa C^{*} C_{\Lambda} \varepsilon\right\rangle_{X}
\end{aligned}
$$

The limits in (23) exist and are finite. Indeed,

$$
\begin{aligned}
& \lim _{\lambda \rightarrow+\infty}\left\langle\varepsilon,-\lambda R(\lambda, A) \kappa C^{*} C_{\Lambda} \varepsilon\right\rangle_{X} \\
&=-\kappa \lim _{\lambda \rightarrow+\infty}\left\langle C \lambda R(\lambda,-A) \varepsilon, C_{\Lambda} \varepsilon\right\rangle_{O}=-\kappa\left\|C_{\Lambda} \varepsilon\right\|_{O}^{2}, \\
& \lim _{\lambda \rightarrow+\infty}\left\langle R(\beta, A) \kappa C^{*} C_{\Lambda} \varepsilon,-\lambda R(\lambda, A) \kappa C^{*} C_{\Lambda} \varepsilon\right\rangle_{X} \\
&=-\kappa^{2} \lim _{\lambda \rightarrow+\infty}\left\langle C \lambda R(\lambda,-A) R(\beta, A) C^{*} C_{\Lambda} \varepsilon, C_{\Lambda} \varepsilon\right\rangle_{O} \\
&=-\kappa^{2}\left\langle G(\beta) C_{\Lambda} \varepsilon, C_{\Lambda} \varepsilon\right\rangle_{O}, \\
&= \lim _{\lambda \rightarrow+\infty}\left\langle R(\beta, A) \kappa C^{*} C_{\Lambda} \varepsilon, \beta R(\beta, A) \lambda R(\lambda, A) \kappa C^{*} C_{\Lambda} \varepsilon\right\rangle_{X} \\
& \kappa^{2} \beta\left\|R(\beta, A) C^{*} C_{\Lambda} \varepsilon\right\|_{X}^{2} .
\end{aligned}
$$


From (23)-(26) we obtain the following identity, $\forall \varepsilon \in D\left(A^{\kappa}\right)$ :

$\left\langle A^{\kappa} \varepsilon, \varepsilon\right\rangle_{X}=-\kappa\left\|C_{\Lambda} \varepsilon\right\|_{O}^{2}-\kappa^{2}\left\langle G(\beta) C_{\Lambda} \varepsilon, C_{\Lambda} \varepsilon\right\rangle_{O}+\kappa^{2} \beta\left\|R(\beta, A) C^{*} C_{\Lambda} \varepsilon\right\|_{X}^{2}$.

Since (27) is true for all $\beta \in \rho(A) \cap \mathbf{R}^{+}$, passing to the limit when $\beta \rightarrow+\infty$, and using $(12),(27)$ can be written as:

$$
\left\langle A^{\kappa} \varepsilon, \varepsilon\right\rangle_{X}=-\kappa\left\|C_{\Lambda} \varepsilon\right\|_{O}^{2}+\lim _{\beta \rightarrow+\infty} \kappa^{2} \beta\left\|R(\beta, A) C^{*} C_{\Lambda} \varepsilon\right\|_{X}^{2} .
$$

The proof of Assertion 3 is complete.

Assertion 4 The errors are exponentially stable if $0<\kappa<1 / K_{\max }$.

Proof of Assertion 4 From Proposition $1, \sqrt{\beta}\left\|R(\beta, A) C^{*} C_{\Lambda} \varepsilon\right\|_{L(O, X)}$ is uniformly bounded for all $\beta>0$. So, the $K_{\max }$ is well defined. On the other hand, by (10):

$$
\lim _{\beta \rightarrow+\infty} \kappa^{2} \beta\left\|R(\beta, A) C^{*} C_{\Lambda} \varepsilon\right\|_{X}^{2} \leq \kappa^{2} K_{\max }\left\|C_{\Lambda} \varepsilon\right\|_{O}^{2} .
$$

From (29) and (16), we obtain:

$$
\begin{gathered}
\frac{1}{2} \frac{d}{d t}\|\varepsilon(t)\|_{X}^{2} \leq-\kappa\left(1-\kappa K_{\max }\right)\left\|C_{\Lambda} \varepsilon\right\|_{O}^{2} \quad \forall \varepsilon \in D\left(A^{\kappa}\right) \Rightarrow \\
\|\varepsilon(t)\|_{X}^{2} \leq\left\|\varepsilon_{0}\right\|_{X}^{2}-2 \kappa\left(1-\kappa K_{\max }\right) \int_{0}^{t}\left\|C_{\Lambda} \varepsilon(\tau)\right\|_{O}^{2} d \tau, \forall t \geq 0 .
\end{gathered}
$$

Since the open loop system is exactly observable, the system (8) is also exactly observable, from Remark 1. By (1), it results that there are $\tilde{\tau}_{0}>0$ and $\tilde{m}>0$ such that:

$$
\int_{0}^{\tilde{\tau}_{0}}\left\|C_{\Lambda} \varepsilon(t)\right\|_{O}^{2} d t \geq \frac{\tilde{m}}{2 \kappa}\left\|\varepsilon_{0}\right\|_{X}^{2}
$$

From (31) and (30), we obtain:

$$
\begin{gathered}
\left\|\varepsilon\left(\tilde{\tau}_{0}\right)\right\|_{X}^{2} \leq\left[1-\tilde{m}\left(1-\kappa K_{\max }\right)\right]\left\|\varepsilon_{0}\right\|_{X}^{2} \Rightarrow \\
\left\|e^{\tau_{0} A^{\kappa}} \varepsilon_{0}\right\|_{X}^{2} \leq(1-\tilde{\tilde{m}})\left\|\varepsilon_{0}\right\|_{X}^{2}, \forall \varepsilon_{0} \in D\left(A^{\kappa}\right),
\end{gathered}
$$

where $\tilde{\tilde{m}}=\tilde{m}\left(1-\kappa K_{\max }\right)$.

From the semigroup properties it results that:

$$
\|\varepsilon(t)\|_{X}^{2} \leq m e^{-r t},
$$

where

$$
\left\|\varepsilon_{0}\right\|_{X}^{2}, m=\sup _{t \in\left[0, \tilde{\tau}_{0}\right]}\left\|e^{t A^{\kappa}}\right\|, r=\tilde{\tau}_{0}^{-1} \ln \left[(1-\tilde{\tilde{m}})^{-1}\right] .
$$


So, the error estimation exponentially tends to 0 on $X$ for all $0<\kappa<1 / K_{\max }$. The proof of Assertion 4 is complete.

Assertion 5 The errors are exponentially unstable if $\kappa>1 / K_{\min }$.

Proof of Assertion 5. From (11) it results that:

$$
\lim _{\beta \rightarrow+\infty} \kappa^{2} \beta\left\|R(\beta, A) C^{*} C_{\Lambda} \varepsilon\right\|_{X}^{2} \geq \kappa^{2} K_{\min }\left\|C_{\Lambda} \varepsilon\right\|_{O}^{2} .
$$

By (16) we obtain:

$$
\frac{1}{2} \frac{d}{d t}\|\varepsilon(t)\|_{X}^{2} \geq \kappa\left(\kappa K_{\min }-1\right)\left\|C_{\Lambda} \varepsilon(t)\right\|_{O}^{2} .
$$

Using (31) on (33), it results that:

$$
\left\|\varepsilon\left(\widetilde{\tau}_{0}\right)\right\|_{X}^{2} \geq\left\{1+\tilde{m}\left(\kappa K_{\min }-1\right)\right\}\left\|\varepsilon_{0}\right\|_{X}^{2},
$$

so

$$
\left\|\varepsilon\left(\widetilde{\tau}_{0}\right)\right\|_{X}^{2} \geq\left(1+\widetilde{\widetilde{m}}^{\prime}\right)\left\|\varepsilon_{0}\right\|_{X}^{2}, \widetilde{\widetilde{m}}^{\prime}=\widetilde{m}\left(\kappa K_{\min }-1\right)
$$

$\widetilde{\widetilde{m}}>0$ if $\kappa>1 / K_{\text {min }}$. If $t \geq 0$, then $t=n \widetilde{\tau}_{0}+\theta$, where $\theta \in\left[0, \widetilde{\tau}_{0}\right)$. Using (34) and the semi - group property, we find:

$$
\left\|e^{t A^{\kappa}} \varepsilon_{0}\right\|_{X}^{2} \geq\left(1+\widetilde{\widetilde{m}}^{\prime}\right)^{n}\left\|e^{\theta A^{\kappa}} \varepsilon_{0}\right\|_{X}^{2} .
$$

Using (34), it results that:

$$
\left(1+\widetilde{\widetilde{m}}^{\prime}\right)\left\|\varepsilon_{0}\right\|_{X}^{2} \leq\left\|e^{\widetilde{\tau}_{0} A^{\kappa}} \varepsilon_{0}\right\|_{X}^{2} \leq \widetilde{K}\left\|e^{\theta A^{\kappa}} \varepsilon_{0}\right\|_{X}^{2},
$$

where $\widetilde{K}=\sup _{t \in\left[0, \widetilde{\tau}_{0}\right]}\left|e^{t A^{\kappa}}\right|_{L(X)}^{2}$. So,

$$
\left\|e^{\theta A^{\kappa}} \varepsilon_{0}\right\|_{X}^{2} \geq\left(1+\widetilde{\widetilde{m}}^{\prime}\right) / \widetilde{K}\left\|\varepsilon_{0}\right\|_{X}^{2}
$$

and from (35):

$$
\left\|e^{t A^{\kappa}} \varepsilon_{0}\right\|_{X}^{2} \geq \widetilde{K}^{-1}\left(1+\widetilde{\widetilde{m}}^{\prime}\right)^{n+1}\left\|\varepsilon_{0}\right\|_{X}^{2} \geq \widetilde{K}^{-1} e^{t \ln \left(1+\widetilde{\widetilde{m}}^{\prime}\right) / \widetilde{\tau}_{0}}\left\|\varepsilon_{0}\right\|_{X}^{2} .
$$

We conclude that $\left\|e^{t A^{\kappa}} \varepsilon_{0}\right\|_{X}$ is exponentially increasing to infinity when $t \rightarrow \infty$. 
Remark 2 The upper limit and $K_{\max }$ are finite, since $\left(A, C^{*}\right)$ is a well posed control system. The $K_{\min }$ is also finite.

If $f \in D(A)$, the lower and upper limits are equal (see (15)). In this case, the conclusion of Theorem 5 remains true, replacing $K_{\max }$ and $K_{\min }$ by:

$$
\begin{aligned}
& K_{\max }=\kappa^{-1}+\kappa^{-2} \sup _{f \in D\left(A^{\kappa}\right),\left|C_{\Lambda} f\right|_{O}=1}\left\langle A^{\kappa} f, f\right\rangle, \\
& K_{\text {min }}=\kappa^{-1}+\kappa^{-2} \inf _{f \in D\left(A^{\kappa}\right),\left|C_{\Lambda} f\right|_{O}=1}\left\langle A^{\kappa} f, f\right\rangle .
\end{aligned}
$$

Note that $K_{\max }$ and $K_{\min }$ don' t depend on $\kappa$ (see the proof of (15)).

Remark 3 Generally, it is not true that $K_{\max }=K_{\min }=0$. To prove this assertion, we consider an example from [21]. Consider the system described by the following equations of partial derivatives on $X=L^{2}(0,1)$ :

$$
\left\{\begin{array}{l}
W_{t}=W_{x} \\
W(0, t)=W(1, t) \\
W(x, 0)=W^{0}(x)
\end{array}\right.
$$

with the observation

$$
y(t)=W(0, t) .
$$

The operator $A=\partial_{x}$ with its corresponding domain of definition is the generator of a $C_{0}$ semigroup on $X$. The observation space is $O=\mathbf{R}$. The observator of the observation $C: X_{1} \rightarrow O$ is such that $C f=f(0)$. It can be prooved that $(A, C)$ is admissible and exactly observable. Moreover, $\left(A, C^{*}, C\right)$ is regular. By Theorem 5 , the Luenberger observer proposed here is governed by the following equation of partial derivatives:

$$
\left\{\begin{array}{l}
\Omega_{t}=\Omega_{x} \\
\Omega(1, t)=\Omega(0, t)-\kappa[\Omega(t, 0)-W(0, t)] \\
\Omega(x, 0)=\Omega^{0}(x) .
\end{array}\right.
$$

It is not difficult to prove that $K_{\max }=K_{\min }=1 / 2$. So, the error of the Luenberger observer, $\epsilon=\Omega-W$, converges to zero if $0<\kappa<2$ and it diverges if $\kappa>2$.

\section{Conclusion}

In this article we built some observers and we found the limits for its exponentially stability, respectively instability. I was also proved that that limits can not be equal to zero, in concordance with the results of [9]. 


\section{Acknowledgment}

The authors are very grateful to Professor Cheng - Zhong Xu, that, by his patience, methodology and exactness created a favourable field for achievement of this work.

\section{References}

[1] Curtain, R. F., Pritchard, A. J., Infinite dimensional linear systems theory, Lecture Notes in Control and Information Sciences, vol. 8, SpringerVerlag, Berlin, 1978.

[2] Curtain, R. F., Weiss, G., Well posedness of triples of operators (in the sense of linear systems theory). Control and estimation of distributed parameter systems (Vorau, 1988), Internat. Ser. Numer. Math., vol.91, Birkhauser, Basel, 1989, pp. 41-59.

[3] Curtain, R. F., Zwart, H., An introduction to infinite-dimensional linear systems theory, Texts in Applied Mathematics, voL. 21, Springer-Verlag, New York, 1995.

[4] Davies, E. B., One-parameter semigroups, Academic Press, London, 1980.

[5] Deguenon, J. Observateurs des Systemes Anti-Adjoints de Dimension Infinie et Application, These unique de doctorat, Universite de Metz, Metz, France, 2003.

[6] Deguenon, A. J., Sallet, G., Xu, C. Z. A Luenberger observer for infinite dimensional skew-symmetric systems with application to an elastic beam, Proc. 2nd Int. Symp on Comm. Control and Signal, Marrakech, 2006.

[7] Jacob, B., Partington J., Admissibility of Control and Observation Operators for Semigroups: A Survey. In: Ball J.A., Helton J.W., Klaus M., Rodman L. (eds) Current Trends in Operator Theory and its Applications. Operator Theory: Advances and Applications, vol 149, Birkhuser, Basel, 2004.

[8] El Jai, A., Pritchard, A.J. Capteurs et actionneurs dans l'analyse des systemes distribues, Masson, New York, 1986, 203 p.

[9] Fuhrmann, P. A. Linear systems and operators in Hilbert space, McGrawHill International Book Co., New York, 1981.

[10] Jurjevic, V., Quinn, J.P. Controlability and stability, Journal of Differential Equations, vol. 28, 1978, pp. 381-389. 
[11] Pazy, A. Semigroups of linear operators and applications to partial differential equations, Springer-Verlag, New York, 1983, 296 p.

[12] Pritchard, A. J., D. Salamon, D., The linear quadratic control problem for infinite-dimensional systems with unbounded input and output operators, SIAM J. Control Optim., vol. 25(1), 1987, pp. 121-144.

[13] Russell, D. L., Weiss, G., A general necessary condition for exact observability, SIAM J. Contr. Optim., vol. 32, 1994, pp. 1-23

[14] Salamon, D., Control and observation of neutral systems, Research Notes in Mathematics, Pitman (Advanced Publishing Program), vol.91, Boston, MA, 1984.

[15] Salamon, D., Infinite-dimensional linear systems with unbounded control and observation: a functional analytic approach, Trans. Amer. Math. Soc., vol. 300(2), 1987, pp. 383-431.

[16] Salamon, D., Realization theory in Hilbert space, Math. Systems Theory, vol. 21,1989 , pp. $147-164$.

[17] Slemrod, M. A note on complete controllability and stabilizability for linear control systems in Hilbert spaces, SIAM J. Contr. Optim., vol. 12, 1974, pp. 500-508.

[18] Weiss, G., The representation of regular linear systems on Hilbert spaces, Control and estimation of distributed parameter systems (Vorau, 1988), Internat. Ser. Numer. Math., vol.91, Birkhauser, Basel, 1989, pp. 367378.

[19] Weiss, G. Regular linear systems with feedback, Mathematics of Control, Signals, and Systems, vol. 7, 1994, pp. 23-57.

[20] Weiss, G. Two conjectures on the admissibility of control operators, International Series of Numerical Mathematics, vol. 100, 1991, pp. 367-378.

[21] Weiss, G., Curtain, F. Dynamic stabilization of regular linear systems, IEEE Transaction Automatic Control, 42, 1997, pp. 4-21.

[22] Weiss, G.,Curtain, R.F. Exponential stabilization of vibrating systems by collocated feedback, Proc. 7th IEEE Mediterranean Symposium on Control and Automation, June 28 - 30, 1999, Haifa, Israel, pp. 1705 1722 . 
[23] Weiss, G., Curtain, R.F., Exponential stabilization of a Rayleigh beam using collocated control, IEEE Trans. Automatic Control, 53, issue 3, 2008 , pp. $643-654$.

[24] Xu, C.Z. Contrôle des systemes hybrides, Polycopie du Cours de DEA, Institut de Mathématiques et de Sciences Physiques, Bénin, 1999.

Judicael DEGUENON,

Université d'Abomey-Calavi,

Ecole Polytechnique d'Abomey-Calavi, Cotonou, Benin

Email: judical.deguenon@epac.uac.bj

Alina BARBULESCU,

Department of Mathematics,

Ovidius University of Constanta,

Bdul Mamaia 124, 900527 Constanta, Romania.

Email: abarbulescu@univ-ovidius.ro 